\title{
Dependence on Dectin-1 Varies With Multiple Candida Species
}

\author{
Aiysha Thompson 1,2, James S. Griffiths' ${ }^{1}$, Louise Walker ${ }^{3}$, Diogo M. da Fonseca', \\ Keunsook K. Lee ${ }^{3}$, Philip R. Taylor ${ }^{1,2}$, Neil A. R. Gow ${ }^{3,4}$ and Selinda J. Orr ${ }^{1+*}$
}

${ }^{1}$ Division of Infection and Immunity and Systems Immunity Research Institute, School of Medicine, Cardiff University, Cardiff, United Kingdom, ${ }^{2}$ UK Dementia Research Institute, Cardiff University, Cardiff, United Kingdom, ${ }^{3}$ Medical Research Council Centre for Medical Mycology, Aberdeen Fungal Group, University of Aberdeen, Aberdeen, United Kingdom, ${ }^{4}$ School of Biosciences, University of Exeter, Exeter, United Kingdom

Four Candida spp. (albicans, glabrata, tropicalis, parapsilosis) cause $>95 \%$ of invasive Candida infections. C. albicans elicits immune responses via pathogen recognition receptors including C-type lectin-like receptors (CLRs). The CLR, Dectin-1 is important for host immunity to $C$. albicans and C. glabrata, however, whether Dectin-1 is important for host defense against $C$. tropicalis or $C$. parapsilosis is unknown. Therefore, we compared the involvement of Dectin-1 in response to these four diverse Candida spp. We found that Dectin-1 mediates innate cytokine responses to these Candida spp. in a species- and cell-dependent manner. Dectin-1 KO mice succumbed to infection with highly virulent $C$. albicans while they mostly survived infection with less virulent Candida spp. However, Dectin-1 KO mice displayed increased fungal burden following infection with each Candida spp. Additionally, T cells from Dectin-1 KO mice displayed enhanced effector functions likely due to the inability of Dectin-1 KO mice to clear the infections. Together, these data indicate that Dectin-1 is important for host defense to multiple Candida spp., although the specific roles for Dectin-1 varies with different Candida spp.

Keywords: Dectin-1, Candida spp., macrophages, dendritic cells, $\mathrm{T}$ cells

\section{INTRODUCTION}

Candida spp. cause $\sim 400,000$ life-threatening invasive infections per year with mortality rates of 46-75\% (Brown et al., 2012). They are the fourth most common pathogens causing hospital acquired infections (HAI) (Hidron et al., 2008; Alangaden, 2011), and they are the most common fungal pathogens causing life-threatening HAIs particularly in immunosuppressed patients (Alangaden, 2011). Candida albicans is the most frequent cause, however, other Candida spp. including C. glabrata, C. tropicalis, and C. parapsilosis are also frequently isolated from patients with invasive candidiasis. In addition, smaller numbers of cases of C. krusei, C. guilliermondii, C. kefyr, C. dubliniensis, and C. zeylanoides infection have been identified (Alangaden, 2011; Karacaer et al., 2014). An emerging multidrug resistant Candida spp., C. auris is now appearing in hospitals across the world and will likely join the top four Candida spp. (C. albicans, C. glabrata, C. tropicalis, and C. parapsilosis) as a major cause of fungal HAIs in the next few years (Chowdhary et al., 2017).

Candida spp. differ in their biology and virulence. C. albicans is polymorphic as it can grow in yeast, pseudohyphal and hyphal forms, however, C. glabrata is monomorphic as it can only grow as blastoconidia (yeast). C. parapsilosis does not form true hyphae but it can grow in yeast form and it can produce psuedohyphae, whereas $C$. tropicalis can grow in yeast form, psuedohyphal form and in 
some reports it has been shown to form hyphae (Fidel et al., 1999; Trofa et al., 2008; Gow et al., 2011; Silva et al., 2012). Multiple studies have linked the formation of C. albicans hyphae with enhanced tissue invasion, damage and virulence (Lo et al., 1997; Gow et al., 2011). In agreement with this, comparison studies in mice have found that $C$. albicans is more pathogenic than any of the other Candida spp. tested (Anaissie et al., 1993; Brieland et al., 2001; Arendrup et al., 2002). Based on in vivo mortality rates and histological changes, Arendrup et al. divided 8 Candida spp. into three groups with decreasing virulence: (1) C. albicans, C. tropicalis, (2) C. glabrata, C. kefyr, C. lusitaniae and (3) C. parapsilosis, C. krusie and C. guilliermondii (Arendrup et al., 2002). According to this study, the top four Candida spp. (C. albicans, C. glabrata, C. tropicalis, and C. parapsilosis) that cause life-threatening HAIs in patients actually display highly different levels of pathogenicity, however, the immune responses induced by these different species have not been comprehensively compared.

The innate immune system is the front line of defense against pathogens including C. albicans. Pathogen associated molecular patterns such as mannans and $ß 1,3$-glucans found in the cell wall of $C$. albicans are recognized by pathogen recognition receptors (PRRs) including C-type lectin-like receptors (CLRs) and Tolllike receptors (TLRs) (Patin et al., 2018). The CLR, Dectin-1, binds 1 1,3-glucans in fungal cell walls. Dectin-1 is important for mediating/regulating immune responses to $C$. albicans and C. glabrata including phagocytosis, cytokine/chemokine production, respiratory burst, inflammatory cell recruitment, neutrophil extracellular traps and T cell responses (Taylor et al., 2007; Branzk et al., 2014; Chen et al., 2017). While Dectin-1 has been shown in many studies to be important for controlling systemic C. albicans infections, the requirement for Dectin-1 is actually fungal strain specific due to differences in chitin levels in their cell walls (Saijo et al., 2007; Marakalala et al., 2013). Dectin$1 \mathrm{KO}$ mice infected with $C$. albicans displayed increased fungal burdens in the kidneys and gastrointestinal tract and the mice succumbed to the infection when infected with C. albicans strains that require Dectin-1 for clearance (Taylor et al., 2007; Marakalala et al., 2013). C. glabrata-infected Dectin-1 KO mice displayed increased fungal burdens in the kidneys and livers, however, they did not succumb to the infection (Chen et al., 2017). While C. tropicalis has been shown to induce Dectin-1 expression (Duan et al., 2018) and inhibition of Dectin-1 in peripheral blood mononuclear cells resulted in reduced C. parapsilosis-induced cytokine production (Toth et al., 2013), the role of Dectin-1 in vivo in response to these Candida spp. is unknown.

In the present study, we examined the role of Dectin1 in response to the four main Candida spp. (C. albicans, C. glabrata, C. tropicalis and C. parapsilosis) responsible for life-threatening HAIs. We found that Dectin-1 mediates innate cytokine responses from macrophages and dendritic cells to these four Candida species in a species and cell dependent manner. We showed that Dectin-1 was required to help clear systemic infections with all four spp. In agreement with previous studies, we found that $C$. albicans was the most pathogenic strain and Dectin-1 KO mice succumbed to infection with C. albicans while they mostly survived infection with the other less pathogenic
Candida spp. In addition, we found that Dectin-1 modulates some anti-fungal $\mathrm{T}$ cell responses. Our study shows that Dectin1 plays an important role in host defense to multiple Candida spp., however, the requirement for Dectin-1 for specific immune responses varies with different Candida spp.

\section{MATERIALS AND METHODS}

\section{Mice}

Dectin-1 KO (Clec7a-/-) (Taylor et al., 2007) and age and gender matched control C57BL/6 mice were maintained and handled according to institutional and UK Home Office regulations.

\section{Ethics Statement}

All procedures were approved by the Cardiff University's Animal Welfare and Ethical Review Body and the UK Home Office. Animal care and use adhered to the Animals (Scientific Procedures) Act 1986 and were performed in accordance with a UK Home Office granted Project License.

\section{Reagents}

GM-CSF and M-CSF (Peprotech), IFN- $\gamma$ and IL-17 ELISAs (R\&D), IL-1 $\beta$, IL-6, IL-10, IL-12p40, and TNF ELISAs and Live/Dead ${ }^{\mathrm{TM}}$ fixable dead cell stain kit (Life Technologies) IFN$\gamma, \mathrm{CD} 4, \mathrm{IL}-17, \mathrm{CD} 3, \mathrm{DX} 5$, and CD8 flow cytometry antibodies and isotype controls (Biolegend) were used in this study. Candida albicans SC5314 was from ATCC. Clinical isolates (Candida albicans AM2005/0463, Candida glabrata SCS74761 and SCSB5311, Candida tropicalis AM2007/0112 and SCS74663, Candida parapsilosis AM2005/0207 and SCSB5882) were a gift from Dr. Donna MacCallum (University of Aberdeen).

\section{Preparation of Candida Cultures}

Candida spp. were plated on YPD agar overnight at $30^{\circ} \mathrm{C}$, then cultured in YPD broth for $\sim 16 \mathrm{~h}$ at $30^{\circ} \mathrm{C}$ with shaking, washed three times with PBS by centrifugation and resuspended at the required concentration for experimentation. For some assays, washed Candida spp. were killed with $100 \mathrm{mM}$ thimerosal (Merck) for $1 \mathrm{~h}$ at room temperature. Thimerosal-killed (TK) Candida spp. were washed three times with PBS to remove excess thimerosal and resuspended at the required concentration for experimentation.

\section{Candida Cell Wall Isolation and Analysis}

Candida spp. were grown overnight in YPD broth, washed and broken up with 20 cycles of $45 \mathrm{~s}$ at $6 \mathrm{rpm} / \mathrm{min}$ using a FastPrep machine (MP Biomedical). The supernatants were centrifuged at $11,000 \mathrm{rpm}$ for $5 \mathrm{~min}$ and the pellet contained the cell wall and debris. The pellets were washed with $1 \mathrm{M} \mathrm{NaCl}$ and then boiled in $50 \mathrm{mM}$ Tris- $\mathrm{HCl}$ buffer $\mathrm{pH} 7.5,2 \%$ SDS, $0.3 \mathrm{M}$ $\beta$-mercaptoethanol and $1 \mathrm{mM}$ EDTA to remove cytoplasmic proteins and membrane proteins. Cell walls were pelleted by centrifugation at 11,000 rpm for 5 min and washed extensively, prior to freeze drying. Acid hydrolysis was then performed on the freeze-dried samples by boiling with trifluoroacetic acid 
for $3 \mathrm{~h}$. Samples were then prepared at a concentration of $0.1 \mathrm{mg} / \mathrm{ml}$ for high pressure liquid chromatography using the CarboPac PA10 column to determine chitin, glucan and mannan levels. $10 \mu \mathrm{l}$ of prepared samples were injected and run through the column at $1.5 \mathrm{ml} / \mathrm{min}$ flow rate with $18 \mathrm{mM}$ sodium hydroxide $(\mathrm{NaOH})$ for $20 \mathrm{~min}$. The column was washed with $200 \mathrm{mM} \mathrm{NaOH}$ and equilibrated with $18 \mathrm{mM} \mathrm{NaOH}$ prior to the next sample. Standards of monomers; glucosamine (for chitin), glucose (for glucan), and mannose (for mannan) were run under the same conditions.

\section{Flow Cytometry Analysis of $\beta 1,3-$ Glucan Exposure on Candida spp.}

$5 \times 10^{6}$ live C. albicans, C. glabrata, C. tropicalis and C. parapsilosis, that had been cultured overnight at $30^{\circ} \mathrm{C}$ in YPD broth, were incubated with $10 \mu \mathrm{g}$ of Dectin-1 Fc, Micl Fc or PBS for $2 \mathrm{~h}$ at $4^{\circ} \mathrm{C}$. Candida cells were washed with PBS. After washing Candida cells were incubated with Pe-conjugated donkey antihuman IgG (Jackson ImmunoResearch) for $30 \mathrm{~min}$. Candida cells were washed with PBS, fixed with BD Cytofix (BD Biosciences) and analyzed on an Attune Flow Cytometer (Invitrogen).

\section{Cell Culture}

Bone marrow from the femurs and tibiae of mice were flushed with PBS. Bone marrow derived macrophages (BMDMs) were generated by culturing cells for 6 days in DMEM containing 10\% heat inactivated fetal bovine serum, 5\% heat inactivated horse serum, $2 \mathrm{mM}$ L-glutamine, $100 \mathrm{U} / \mathrm{ml}$ penicillin/streptomycin, $10 \mathrm{mM}$ HEPES and 10ng/ml M-CSF (Patin et al., 2016). BMDCs were generated by culturing cells for 8 days in RPMI 1640 medium containing $10 \%$ heat inactivated fetal bovine serum, $2 \mathrm{mM}$ L-glutamine, $100 \mathrm{U} / \mathrm{ml}$ penicillin/streptomycin, $10 \mathrm{mM}$ HEPES, 1\% NEAA, $1 \mathrm{mM}$ Sodium pyruvate, $50 \mu \mathrm{M}$ $\beta$-mercaptoethanol and $10 \mathrm{ng} / \mathrm{ml}$ GM-CSF.

\section{Cell Stimulations and Cytokine Assays}

BMDMs were harvested using $8 \mathrm{mg} / \mathrm{ml}$ lidocaine and resuspended in RPMI 1640 containing 10\% heat inactivated fetal bovine serum and $100 \mathrm{U} / \mathrm{ml}$ penicillin/streptomycin. BMDMs and BMDCs were plated at a density of $1 \times 10^{5}$ cells/well of a 96-well plate and left overnight at $37^{\circ} \mathrm{C}$. Media was removed, and cells were stimulated with $1 \times 10^{5}$ Candida CFUs/well for $24 \mathrm{~h}$ in a total of $200 \mu \mathrm{l}$ fresh media. Fungizone $(2.5 \mu \mathrm{g} / \mathrm{ml})$ was added $2 \mathrm{~h}$ after stimulation. Cell culture supernatants were recovered and assayed for cytokine by ELISA, according to the manufacturer's protocol.

\section{In vivo Candida spp. Infections}

Mice were injected intravenously with $100 \mu \mathrm{l}$ of Candida spp. in PBS. The fungal load inoculated to the mice for specific experiments are outlined in the figure legends. Mice were monitored using a predefined scoring system and weighed daily, with loss of $20 \%$ body weight being an additional endpoint. At the end of the experiment, serum, kidneys, brains and spleens were harvested as previously described (Patin et al., 2016). Mice were sacrificed by $\mathrm{CO}_{2}$ administration and cervical dislocation. Mice were bled by cardiac puncture and organs were harvested. Serum was centrifuged at $10,000 \mathrm{rpm}$ for $10 \mathrm{~min}$ at $4^{\circ} \mathrm{C}$ in serum tubes and assayed for cytokine by ELISA. The left kidney and right brain were placed in PBS, homogenized and serial dilutions were plated on YPD plates containing $50 \mu \mathrm{g} / \mathrm{ml}$ chloramphenicol, incubated at $30^{\circ} \mathrm{C}$ for $24-48 \mathrm{~h}$ and the colonies were counted per gram organ. The spleen was homogenized and red blood cells were lysed using ACK lysis buffer. Cells were washed and resuspended in IMDM containing $10 \%$ heat inactivated fetal bovine serum, $2 \mathrm{mM}$ L-glutamine, $100 \mathrm{U} / \mathrm{ml}$ penicillin/streptomycin, $50 \mu \mathrm{M} \beta$-mercaptoethanol. Splenocytes were plated at $1 \times 10^{6}$ cells/well and stimulated with $2 \times 10^{6} \mathrm{CFU} /$ wells Candida spp. for $48 \mathrm{~h}$ at $37^{\circ} \mathrm{C}$. Fungizone $(2.5 \mu \mathrm{g} / \mathrm{ml})$ was added $2 \mathrm{~h}$ after stimulation. After $48 \mathrm{~h}$, supernatants were recovered and IFN- $\gamma$ and IL-17 levels were measured by ELISA. Splenocytes were plated at $1 \times 10^{6}$ cells/well and stimulated with PMA/Ionomycin for $4 \mathrm{~h}$ at $37^{\circ} \mathrm{C}$ in the presence of Brefeldin A. IFN- $\gamma$ and IL-17 producing T-cells were determined by flow cytometry.

\section{T Cell Flow Cytometry}

Following PMA/Ionomycin stimulation, splenocytes were washed with PBS, and stained with live/dead fixable stain (Thermo Fisher Scientific) for $15 \mathrm{~min}$ at $4^{\circ} \mathrm{C}$. Cells were washed $1 \times$ with PBS and $1 \times$ with FACS buffer. Cells were blocked with $4 \mathrm{mg} / \mathrm{ml} 2.4 \mathrm{G} 2$ in 5\% rabbit serum in FACS buffer for $15 \mathrm{~min}$ at $4^{\circ} \mathrm{C}$. Cells were stained with CD8, CD4, CD3, NK1.1 antibodies (Biolegend) in FACS buffer for $30 \mathrm{~min}$ at $4^{\circ} \mathrm{C}$. Cells were washed with FACS buffer, incubated with $\mathrm{BD}$ cytofix/cytoperm buffer (BD Biosciences) for $20 \mathrm{~min}$ at $4^{\circ} \mathrm{C}$. Cells were washed with BD Permwash buffer (BD Biosciences). Cells were blocked with $4 \mathrm{mg} / \mathrm{ml} \mathrm{2.4G2}$ in 5\% rabbit serum in FACS buffer for $15 \mathrm{~min}$ at $4^{\circ} \mathrm{C}$. Cells were stained with IFN- $\gamma$ and IL17 antibodies for $30 \mathrm{~min}$ at $4^{\circ} \mathrm{C}$. Cells were washed with $\mathrm{BD}$ Permwash and analyzed on a Cyan flow cytometer (Beckman Coulter) or a BD FACS Canto flow cytometer. Data was analyzed using FlowJo software.

\section{Statistical Methods}

Data were analyzed using GraphPad Prism. All data are presented as means \pm SEM. Student's $t$ test was used for statistical analysis for two groups or Bonferonni's post-test after Two-way ANOVA for multiple groups. When data did not follow a Gaussian distribution, it was transformed by $Y=\operatorname{sqrt}(Y+0.5)$ and analyzed by Student's $t$ test/ANOVA or non-parametric tests if data still did not follow a Gaussian distribution. $p$ values less than 0.05 were considered statistically significant: ${ }^{*} p<0.05$, ** $p<0.005,{ }^{* * *} p<0.001$.

\section{RESULTS}

\section{Candida spp. Display Differences in Their Cell Wall Composition}

As Dectin-1 is important for the immune response to C. albicans and C. glabrata (Taylor et al., 2007; Marakalala et al., 2013; 
Chen et al., 2017) we wanted to determine whether it plays a role in response to other medically relevant Candida spp. To this end we performed compositional analysis by HPLC of the three major polysaccharides (glucan, mannan, and chitin) in the cell wall of four Candida spp. (albicans, glabrata, tropicalis, and parapsilosis). The strains of Candida used for this analysis were the common C. albicans SC5314 lab strain while the C. glabrata SCS74761, C. tropicalis SCS74663 and C. parapsilosis SCSB5882 strains were clinical isolates. Glucan levels were highest in $C$. glabrata, followed by C. albicans, C. tropicalis, and C. parapsilosis (Table 1). The opposite was true for mannan levels as these were highest in C. parapsilosis followed by C. tropicalis, C. albicans and C. glabrata (Table 1). Chitin levels were highest in C. tropicalis, followed by C. glabrata, C. albicans, and C. parapsilosis (Table 1). As high levels of glucan are present in the cell wall of each of these different Candida spp., Dectin-1 will likely be involved in the immune response to these clinically relevant Candida spp.

\section{Candida spp. Display Differential 11,3-Glucan Exposure}

While Dectin-1 binds $\beta 1,3$-glucan in fungal cell walls (Brown and Gordon, 2001), an important factor in determining Dectin-1 binding is the level of $\beta 1,3$-glucan exposure (Ballou et al., 2016; Chen et al., 2017). As we observed compositional differences in glucan levels in the four Candida spp. (Table 1), we next aimed to determine whether these Candida spp. displayed different levels of $\beta 1,3$-glucan exposure. To this end we stained Candida cells cultured in YPD with Dectin-1 Fc or with Micl Fc as a control and quantified mean fluorescence intensity (MFI) by flow cytometry. In agreement with the compositional analysis, C. glabrata displayed the highest level of $\beta 1,3$-glucan exposure (Figures 1A,B). However, C. albicans displayed the lowest level of $\beta 1,3$-glucan exposure, which differs from the compositional analysis, while C. tropicalis and C. parapsilosis displayed an intermediate level of $\beta 1,3$-glucan exposure (Figures 1A,B). The four Candida spp. tested all have some exposed $\beta 1,3$-glucan on their cell wall suggesting that Dectin-1 will be involved in the immune response to each of these species, however, differences in the level of $\beta 1,3$-glucan exposure between the species may control the level of Dectin-1 involvement in various responses.

\section{Dectin-1 Mediates Cytokine Responses to Multiple Candida spp.}

Dectin-1 has been shown to mediate C. albicans- and C. glabratainduced cytokine production from various myeloid cell populations (Taylor et al., 2007; Chen et al., 2017). Here, we compared the requirement for Dectin-1 to mediate cytokine production in response to four clinically relevant Candida spp. To investigate this, BMDCs and BMDMs from WT and Dectin-1 KO mice were stimulated with C. albicans SC5314, C. glabrata SCS74761, C. tropicalis SCS74663 or C. parapsilosis SCSB5882. Interestingly, the Candida spp. induced rather different cytokine profiles and this varied between BMDCs and BMDMs (Figures 2A,B). We found that TNF production in response to all Candida spp. was partially dependent on Dectin1 in BMDCs and BMDMs (Figures 2A,B). C. parapsilosisinduced IL-12p40 production in BMDMs was largely dependent on Dectin-1 (Figure 2B) while it was partially dependent on Dectin-1 in BMDCs in response to all Candida spp. (Figure 2A). Candida-induced IL- $1 \beta$ production was independent of Dectin1 (Figures 2A,B). IL-6 production in BMDCs was partially dependent on Dectin-1 while it was independent of Dectin1 in BMDMs (Figures 2A,B). C. albicans induced the highest levels of IL-10 in both BMDMs and BMDCs compared to other Candida spp. In BMDCs, IL-10 production was partially dependent on Dectin-1 while it was independent of Dectin1 in BMDMs (Figures 2A,B). Results were largely similar with an additional strain for each Candida spp. (C. albicans AM2005/0463, C. glabrata SCSB5311, C. tropicalis AM2007/0112 or C. parapsilosis AM2005/0207) in BMDMs (Figure 2C). In particular, similar to the results in Figure 2B, we found that TNF production in response to all Candida spp. was partially dependent on Dectin-1 and C. parapsilosis-induced IL-12p40 production in BMDMs was largely dependent on Dectin-1 using additional strains of these 4 Candida spp. (Figure 2C). We then killed the Candida strains used in Figures 2A,B with thimerosal, which maintains cell wall integrity (Hall et al., 2013), and stimulated WT and Dectin-1 KO BMDMs at an MOI of 1 for $24 \mathrm{~h}$, similar to Figures 2A-C. We found that the thimerosal-killed Candida spp. induced much lower cytokine levels (Figure 2D) than live Candida (Figure 2B) and in some cases, cytokine production was not detected (nd). Using the thimerosal-killed Candida spp., we did not detect significant dependence on Dectin-1 for cytokine production, although as mentioned above cytokine production was substantially lower than that induced by live Candida spp. In addition to cytokine production, nitric oxide production is an important immune response to fungal pathogens. We found that C. tropicalis and C. parapsilosis induced higher levels of nitric oxide in BMDMs compared to C. albicans and C. glabrata, while all Candida spp. induced

TABLE 1 | Relative proportions of carbohydrates in the cell wall extracted from different Candida spp.

\begin{tabular}{|c|c|c|c|}
\hline Candida species & Chitin & Glucan & Mannan \\
\hline C. albicans SC5314 & $1.72( \pm 0.08)$ & $62.97( \pm 1.1)$ & $35.31( \pm 1.15)$ \\
\hline C. glabrata SCS74761 & $2.40( \pm 0.14)$ & $69.51( \pm 0.97)^{* * *}$ & $28.09( \pm 1.04)^{* * *}$ \\
\hline C. tropicalis SCS74663 & $3.24( \pm 0.49)$ & $59.29( \pm 0.42)^{* * \# \# \#}$ & $37.47( \pm 0.38)^{\# \# \#}$ \\
\hline C. parapsilosis SCSB5882 & $0.37( \pm 0.01)^{€}$ & $40.75( \pm 0.3)^{* * * \# \# \# € € €}$ & 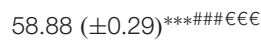 \\
\hline
\end{tabular}

${ }^{*} p<0.05,{ }^{* *} p<0.01,{ }^{* * *} p<0.001 .{ }^{*}$ compared to C. albicans, ${ }^{*}$ compared to C. glabrata, ${ }^{€}$ compared to C. tropicalis. 


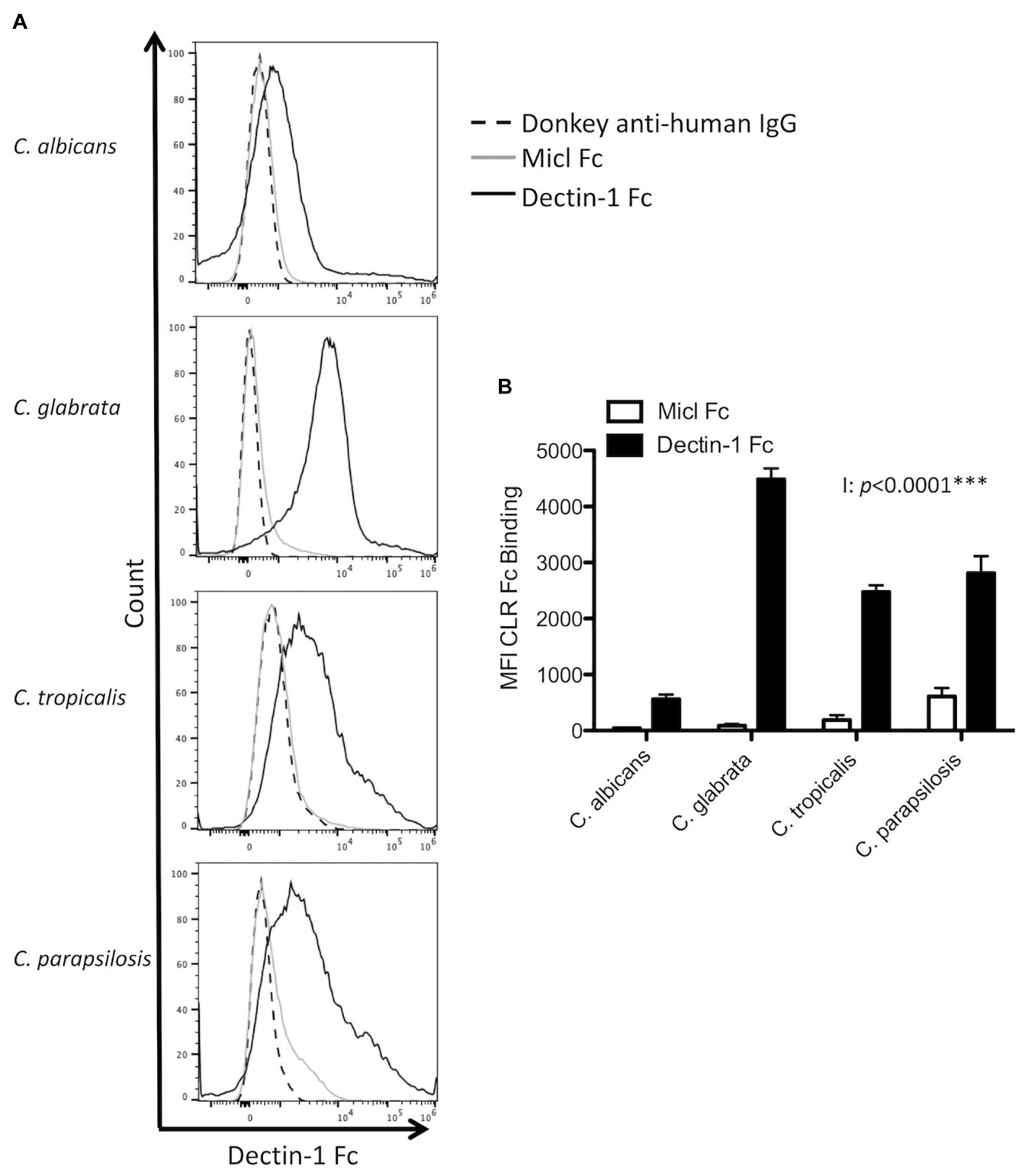

FIGURE 1 | Candida spp. display differential $\beta 1,3$-glucan exposure. (A,B) C. albicans SC5314, C. glabrata SCS74761, C. tropicalis SCS74663 and C. parapsilosis SCSB5882 were incubated with Dectin-1 Fc, Micl Fc or PBS followed by incubation with Pe-conjugated Donkey anti-human IgG. $\beta 1,3$-glucan exposure on the Candida cell surface was analyzed by flow cytometry. (A) Representative flow plots display $\beta 1,3-$ glucan exposure from 3 independent experiments. (B) Graphs display MFI of control Micl Fc or Dectin-1 Fc minus MFI of Pe-conjugated Donkey anti-human IgG. Graphs display cumulative data from 3 independent experiments. 2-way ANOVA with Bonferroni's post-test I = Interaction between Candida spp. and CLR Fc binding.

similar levels of nitric oxide in BMDCs. However, nitric oxide production was independent of Dectin-1 in both BMDMs and BMDCs (Figures 2E,F). Thus, Candida spp. induce different cytokine profiles to each other and Dectin-1 mediates Candidainduced cytokine responses to live Candida spp. but not nitric oxide production, in a cytokine-, cell type-, and Candidadependent manner.

\section{Dectin-1 Mediates Candida Clearance}

As Dectin-1 mediates Candida-induced cytokine production (Figure 2), we next compared the role for Dectin-1 in controlling systemic infection with these different Candida spp. We first performed an experiment to determine which dose of each Candida spp. to use during in vivo experiments. Fungal burden in the kidneys of Candida-infected mice was compared to mice infected with $1.5 \times 10^{5} \mathrm{CFU}$ C. albicans. A dose that caused similar kidney fungal burdens was selected for the other strains (Supplementary Figure 1). WT and Dectin$1 \mathrm{KO}$ mice were infected with four Candida spp. and in agreement with previous findings (Arendrup et al., 2002), C. tropicalis, C. glabrata, and C. parapsilosis were less virulent than C. albicans. Several WT and Dectin-1 KO mice succumbed 


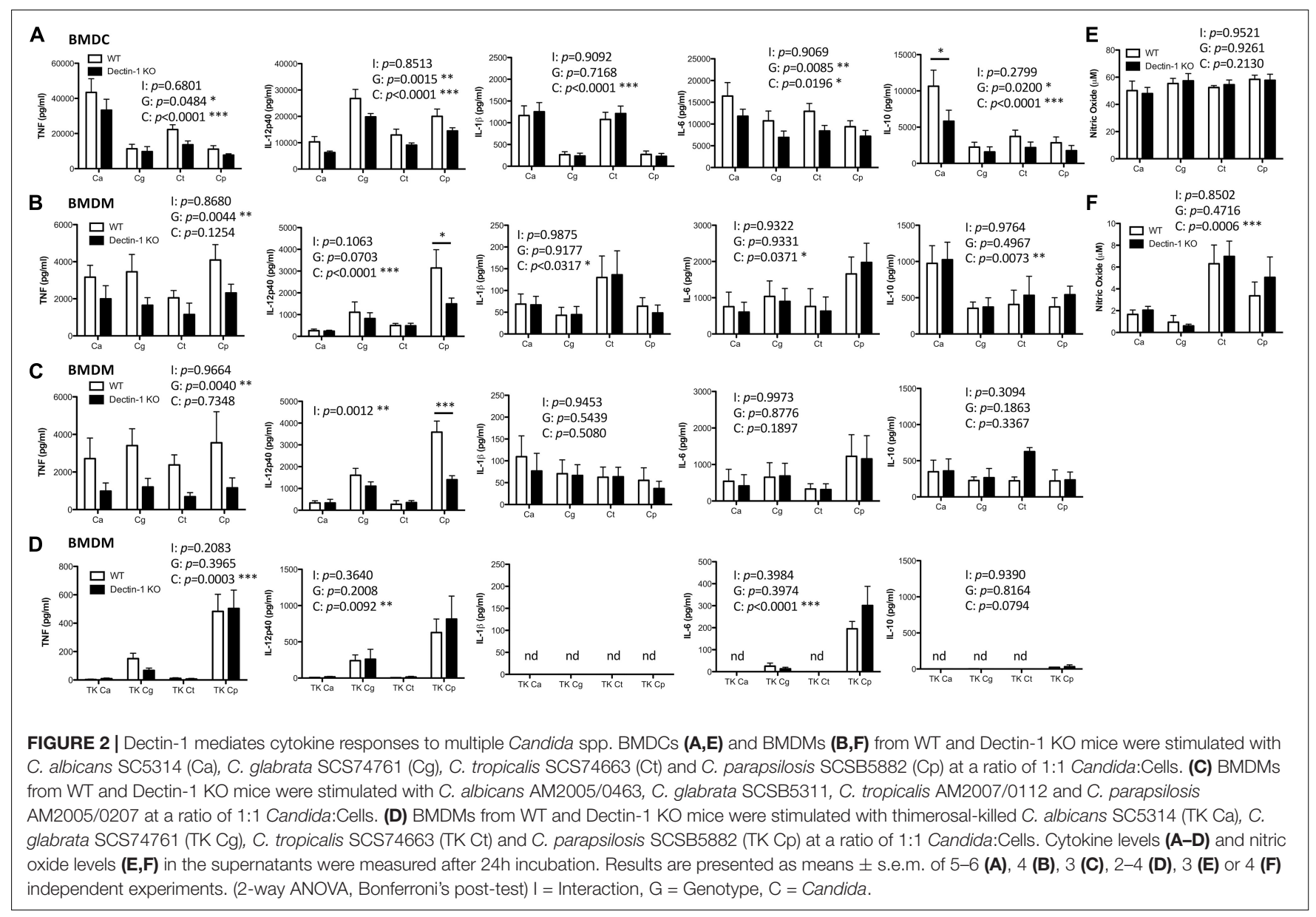

to infection with $C$. albicans, and Dectin-1 KO mice were more susceptible than WT mice (Figure 3A), similar to previous findings (Taylor et al., 2007). However, mice infected with the other Candida spp. (Figure 3A) largely did not succumb to infection even though they were infected with $\approx 6.6-100 \times$ more CFU than C. albicans. Dectin-1 KO mice trended toward slightly increased fungal burden in the kidneys and/or brains compared to WT mice at these timepoints (20-30 days) (Figure 3B), although statistically significant increases were not identified at these timepoints for infection with most of the Candida spp., We then investigated the importance of Dectin-1 during the early stages of infection. Since many of the $C$. albicans-infected mice succumbed to infection within 1 week (Figure 3A), mice were infected with 10x less C. albicans for Figures 4A,B. One week following infection with all four Candida spp. Dectin-1 KO mice displayed increased fungal burden in the kidneys and/or brains compared to WT mice (Figure 4A). In addition, C. albicans-infected Dectin-1 KO mice displayed increased serum IL-12p40 and IL6 levels (Figure 4B), likely due to their inability to control the infection. We observed limited TNF, IL-1 $\beta$, and IL-10 levels in the serum of these mice. Serum cytokine levels in response to other Candida spp. were not significantly different in Dectin-1 KO mice (Figure 4B). These data indicate that
Dectin-1 is important for surviving infection with the highly virulent C. albicans, while it is dispensable for surviving infection with less virulent spp. However, Dectin-1 mediates fungal clearance of multiple Candida spp. particularly at early stages of infection.

\section{Dectin-1 Regulates Candida-Associated T Cell Responses}

While Dectin-1 is mainly recognized as an important mediator of innate anti-fungal responses, it has also been shown to regulate T cell responses (Robinson et al., 2009; Chen et al., 2017). Therefore, we examined the role of Dectin-1 during $\mathrm{T}$ cell responses to four Candida spp. 7 days post-infection, splenic Ifng and Tbx21 mRNA levels of all Candida-infected mice were similar between WT and Dectin-1 KO mice, however, Il17 and Rorc mRNA levels were increased in C. albicans-infected Dectin$1 \mathrm{KO}$ mice compared to WT mice. No major differences were observed in Il17 levels (low or undetected) or Rorc levels in mice infected with the other Candida spp. (Figure 5A). We next examined the potential of splenic $\mathrm{T}$ cells from these infected mice to produce IFN- $\gamma$ and IL-17 following PMA/Ionomycin stimulation. This mainly induced a Th1 response with a much smaller Th17 response. IFN- $\gamma$ producing T cells were somewhat 
A

C. albicans

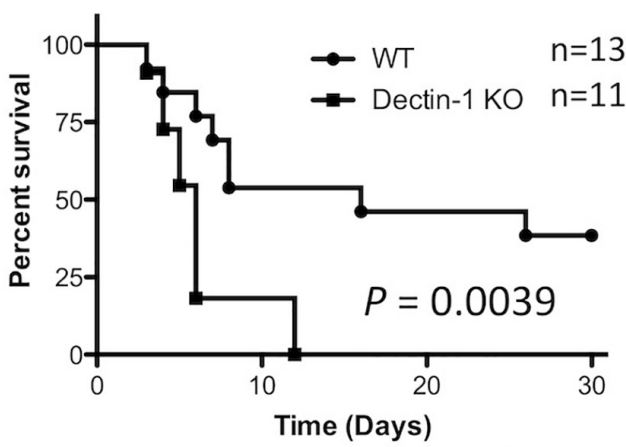

C. glabrata

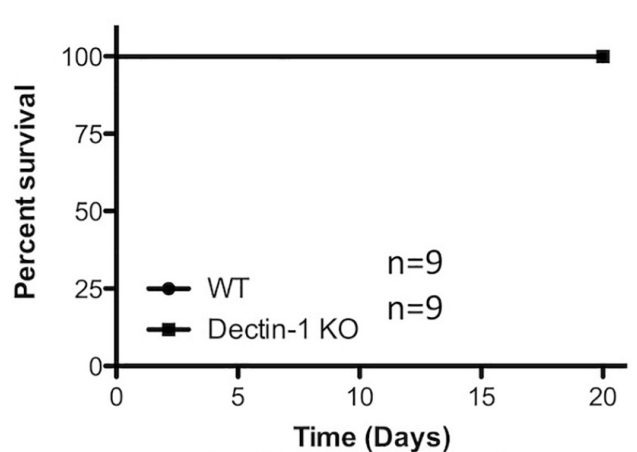

C. tropicalis

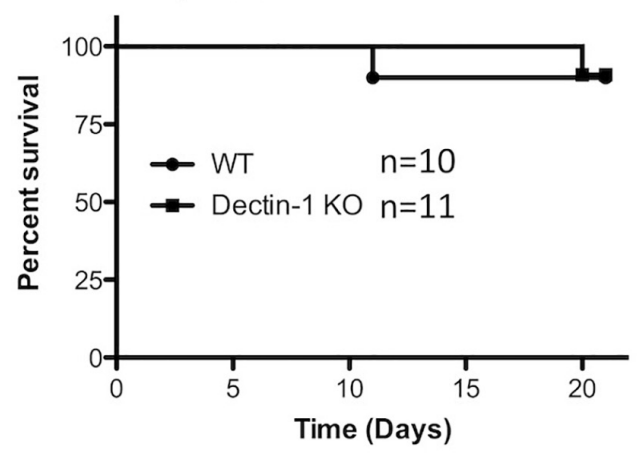

C. parapsilosis $1.5 \times 10^{7}$

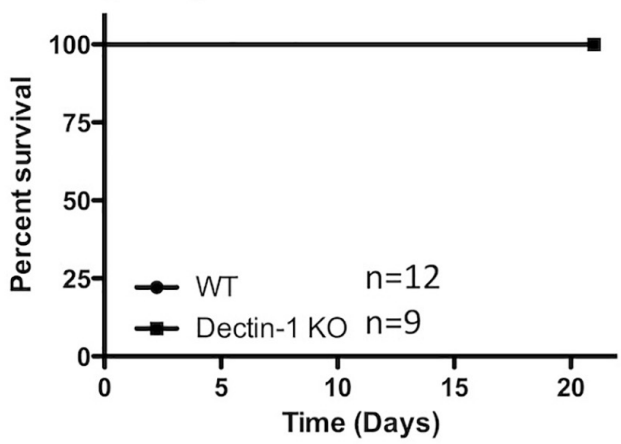

B

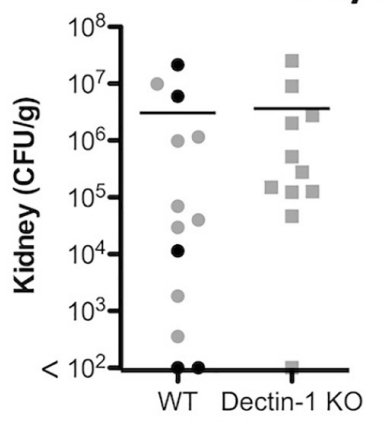

30: $1.5 \times 10^{5}$

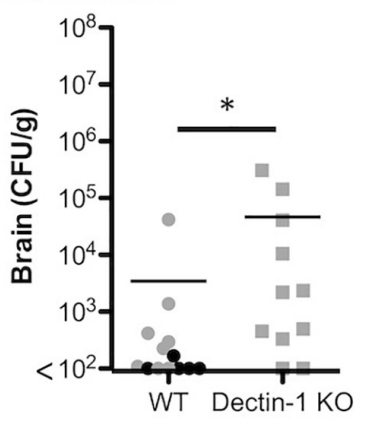

Day 20: $1.5 \times 10^{7}$
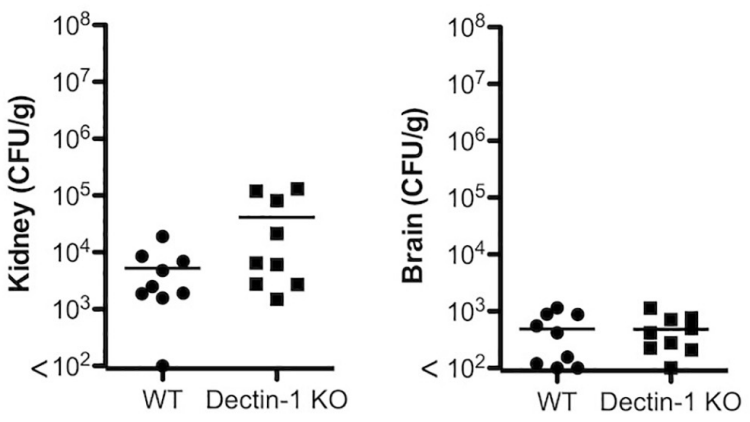

Day 21: $1 \times 10^{6}$
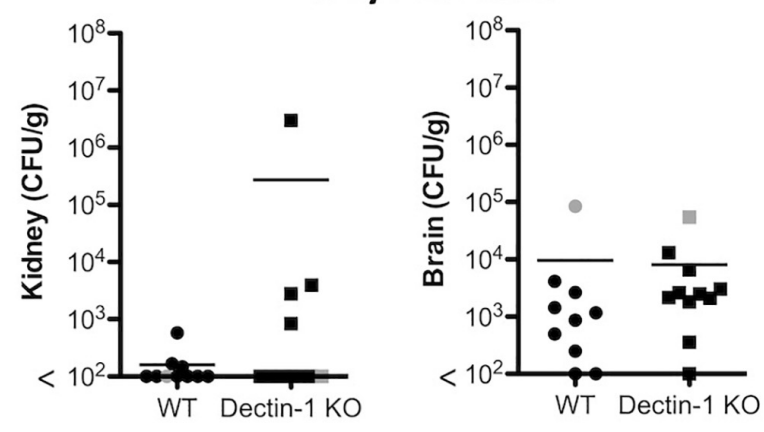

Day 21: $1.5 \times 10^{7}$

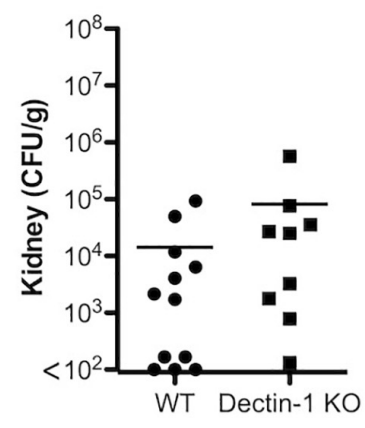

FIGURE 3 | Dectin-1 KO mice are susceptible to infection with highly virulent $C$. albicans. (A) WT and Dectin-1 KO mice were infected intravenously with the indicated doses of $C$. albicans for 30 days, C. glabrata for 20 days, C. tropicalis for 21 days or C. parapsilosis for 21 days. Survival curves based on humane end-point of infected WT (filled circles) and Dectin-1 KO mice (filled squares). Graphs are the cumulative result of 2 independent experiments. Log-rank test. (B) CFU in the kidneys at 20-30 days after infection (black symbols) or at time of death by humane end point (gray symbols). Graphs are the cumulative result of 2

independent experiments. Each symbol represents an individual mouse. Student's $t$ test on transformed data. 


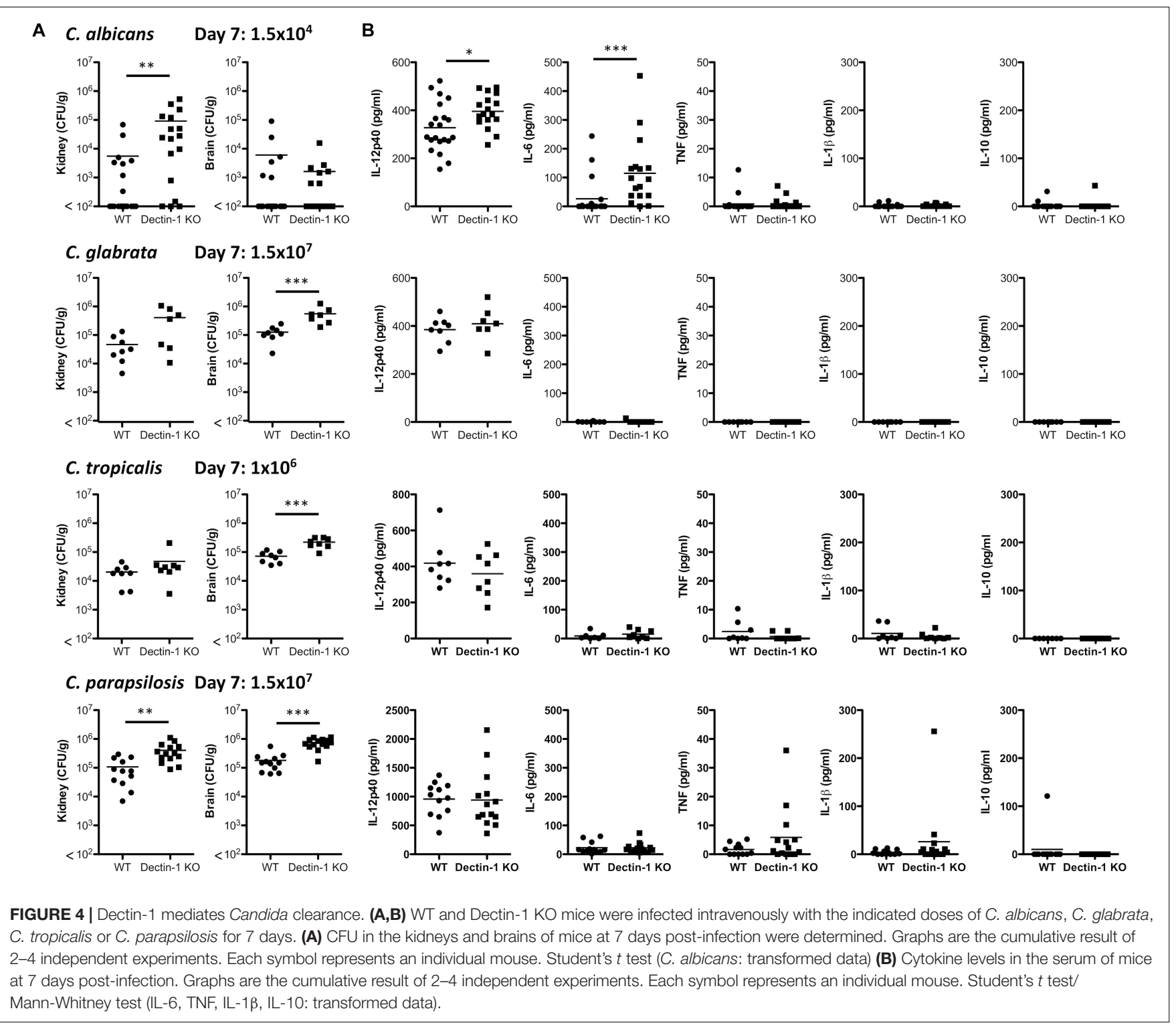

reduced in spleens from C. glabrata-infected Dectin-1 KO mice following restimulation with PMA/Ionomycin (Figure 5B and Supplementary Figure 2), while T cells from C. albicans- and C. parapsilosis-infected Dectin-1 KO mice displayed increased IL-17 production following stimulation with PMA/Ionomycin (Figure 5B). Finally, upon antigen restimulation with live Candida spp. in the presence of fungizone, Dectin-1 KO splenocytes displayed increased IL-17 production in response to C. albicans (significant) and C. parapsilosis (trend) but not C. glabrata or C. tropicalis (Figure 5C). Similar to our observations with splenic Il17 mRNA levels, C. albicans induced the highest levels of IL-17 upon restimulation compared to other Candida spp. Dectin-1 KO splenocytes also displayed enhanced IFN- $\gamma$ production following restimulation with $C$. albicans but not with other Candida spp. These data indicate that Dectin1 regulates Candida-associated $\mathrm{T}$ cell responses, in addition to myeloid cell responses.

\section{DISCUSSION}

Here, we have shown that four clinically relevant Candida spp. display differences in their polysaccharide composition and $\beta 1,3$ glucan exposure. We found that C. glabrata contained the highest glucan proportion and displayed the most $\beta 1,3$-glucan exposure, however, we did not find that glucan composition and $\beta 1,3$ glucan exposure necessarily correlated for the other Candida spp. The 4 Candida spp. displayed some level of $\beta 1,3$-glucan exposure and cytokine production in response to all four Candida spp. was partially dependent on Dectin-1, however, this varied with Candida spp. and cell type. During systemic infections with the various Candida spp. we found, similar to others, that C. albicans was the most virulent Candida spp. out of the four spp. used. Similar to C. albicans, we showed that Dectin-1 is important for early clearance of various Candida spp. In addition, we found that Dectin-1 regulates some Candida-specific T cell responses. 
A

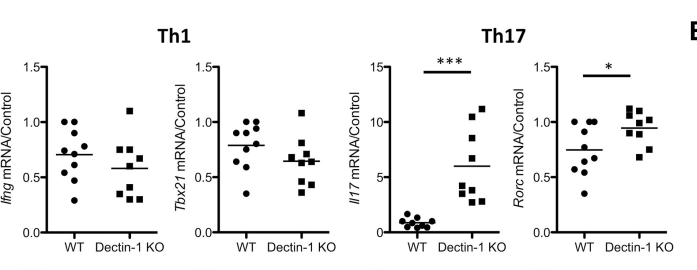

C. glabrata
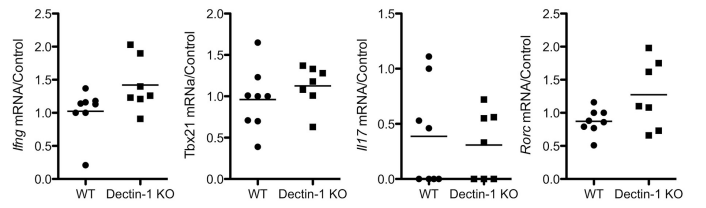

$\underbrace{}_{\text {WT Dectin-1 KO }} \underbrace{1.5}_{0.0} \underbrace{1.5}_{\text {WT Dectin- } 1 \text { KO }}$
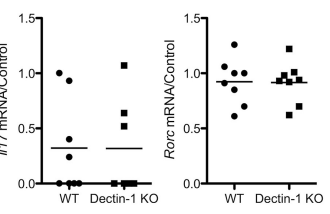

C. parapsilosis

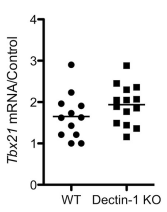

B
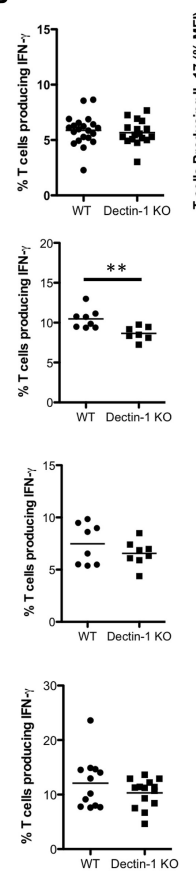

C
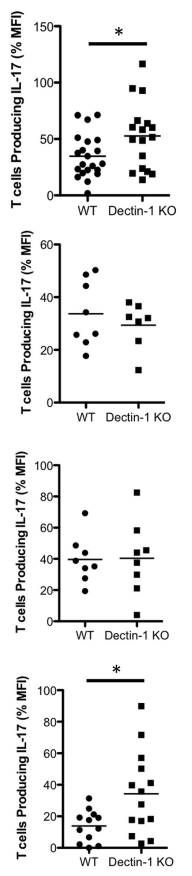
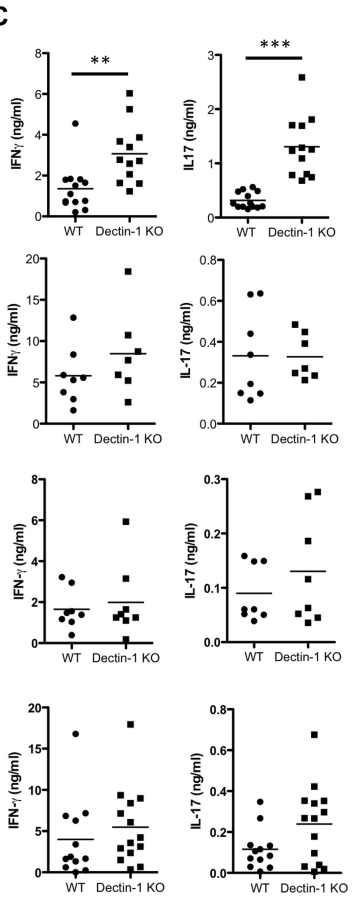

FIGURE 5 | Dectin-1 regulates Candida-associated T cell responses. (A-C) WT and Dectin-1 KO mice were intravenously infected with $1.5 \times 10^{4}$ CFU C. albicans, $1.5 \times 10^{7}$ CFU C. glabrata, $1 \times 10^{6}$ CFU C. tropicalis or $1.5 \times 10^{7}$ CFU C. parapsilosis for 7 days. (A) RNA was isolated from the spleens of infected mice, cDNA was prepared and mRNA transcripts were detected by real-time qPCR. mRNA levels were normalized to Hprt1. Graphs display the cumulative result of 2 independent experiments. Each symbol represents one mouse. (Student's $t$ test) (B) Splenocytes from infected mice were restimulated with PMA/lonomycin for $4 \mathrm{~h}$ and IFN- $\gamma$ and IL-17 levels were measured by FACS. Graphs display\% T cells producing IFN- $\gamma$ - Isotype or MFI of IL-17 production by T cells. IL-17 MFI by cells from one WT mouse stimulated with PMA/lonomycin was set at $100 \%$ and all other IL-17 MFI in that experiment are presented as a\% of this. MFI for the Isotype was then subtracted. (C) Splenocytes from infected mice were restimulated with live Candida spp. for $48 \mathrm{~h}$ and IFN- $\gamma$ and IL-17 levels were measured by ELISA. (A-C) Graphs are the cumulative result of 2-4 independent experiments. Each symbol represents an individual mouse. Student's $t$ test/Mann-Whitney test.

Overall, we found that while Dectin-1 mediates/regulates various immune responses to different Candida spp., the importance of Dectin-1 for specific immune responses varies for each Candida spp.

For this study, we hypothesized that different responses induced by these four clinically relevant Candida spp. could potentially be due to the availability of ligands in their cell wall. Analysis of the polysaccharide composition and $\beta 1,3$ glucan exposure of these four spp. revealed significant differences between the spp. We found that $C$. glabrata displayed the highest $\beta 1,3$-glucan exposure, C. tropicalis and C. parapsilosis displayed intermediate exposure and $C$. albicans displayed the lowest level of $\beta 1,3$-glucan exposure. Apart from C. glabrata, there did not appear to be any correlation between glucan level in the cell wall and $\beta 1,3$-glucan exposure. As the cell wall analysis detects glucan and does not differentiate between $\beta 1,3$-glucan or $\beta 1,6$-glucan, this could potentially explain the lack of correlation between the level of glucan in the cell wall and the level of $\beta 1,3$-glucan exposure or Dectin-1 binding. Regardless of the level of $\beta 1,3$ glucan exposure, all four spp. displayed some dependence on Dectin-1 for various immune responses, however, we did not observe any clear correlation of the level of glucan in the cell wall or the level of $\beta 1,3$-glucan exposure with dependence on Dectin1. Further analysis of multiple strains of each Candida species would be required to determine whether the differences in cell wall composition or ligand availability are reflective of the species or of a particular strain. In fact, various groups have previously examined polysaccharide composition and $\beta 1,3$-glucan exposure on some of these Candida spp. and these results sometimes differ from ours and from each other (Linden et al., 2010; EstradaMata et al., 2015; Sem et al., 2016). For example, one group showed that $C$. parapsilosis contains a higher \% glucan content that $C$. albicans, which differs from our results (Estrada-Mata et al., 2015). However, another group showed that $C$. parapsilosis displays more $\beta 1,3$-glucan exposure than $C$. albicans, which is in agreement with our results (Linden et al., 2010). In addition, another group showed that $\beta 1,3$-glucan exposure increased from C. tropicalis, C. albicans, C. glabrata to C. parapsilosis (Sem et al., 2016). These data suggest that glucan composition and $\beta 1,3$-glucan exposure vary from one Candida strain to another and these differences are not necessarily reflective of Candida spp. rather each individual strain. Differences in experimental conditions and Candida strains between research groups could potentially explain these different results. We and others have examined $\beta 1,3$-glucan exposure at a particular point, usually after overnight culture in YPD broth. These Candida spp. differ in their ability to form yeast, pseudohyphae or hyphae (Gow et al., 2011; Silva et al., 2012), and in an in vitro assay and in vivo 
these Candida spp. will undergo changes that will modify the level of $\beta 1,3$-glucan exposure over time. In fact, Ballou et al. (2016) showed that lactate in the growth media masks $\beta 1,3$ glucan compared to glucose in the growth media. In addition, $\beta 1,3$-glucan, chitin and/or mannan exposure can be affected by the addition of neutrophils and/or normoxic versus anoxic culture conditions (Lopes et al., 2018). Furthermore, Marakalala et al. (2013) showed that Dectin-1 dependence in vivo was determined by changes in the fungal cell wall chitin content that occurred in vivo. Therefore, many factors will influence $\beta 1,3$ glucan exposure and Dectin-1 dependence over time for various immune responses, so it is not surprising that we did not observe direct correlations between the level of $\beta 1,3$-glucan exposure at one particular timepoint with the level of Dectin-1 involvement during various anti-fungal responses.

In this study, we assessed the involvement of Dectin-1 in mediating Candida-induced cytokine production from BMDCs and BMDMs. In general, we found that the Candida spp. induced much higher levels of cytokines from BMDCs than from BMDMs. Similar to previous findings with C. albicans (Gow et al., 2007; Taylor et al., 2007; Chen et al., 2017), we found that Dectin-1 partially mediates TNF production to the various Candida spp. in both BMDCs and BMDMs. We also found that Dectin-1 partially mediates IL-12p40 production from BMDCs in response to various Candida spp. and it largely mediates IL12 p40 production in response to C. parapsilosis from BMDMs. In addition, Dectin-1 somewhat contributes to C. glabrata-induced IL-12p40 production by BMDMs similar to previous findings (Chen et al., 2017). IL-6 and IL-10 production in response to the various Candida spp. was partially dependent on Dectin1 in BMDCs but not BMDMs. Various groups have shown that Dectin-1 mediates cytokine production such as TNF, IL-6, IL-12p20 and IL-10 from monocytes, macrophages or DCs to Candida spp., zymosan or curdlan (Gow et al., 2007; LeibundGutLandmann et al., 2007; Taylor et al., 2007; Estrada-Mata et al., 2015; Chen et al., 2017), whereas others have shown that Dectin1 is dispensable for these responses in macrophages (Saijo et al., 2007) or BMDCs (Robinson et al., 2009). Several factors could contribute to the different results observed including the source of the macrophages (peritoneal, alveolar, BMDM), cell culture conditions, type of ligand used (live/killed Candida spp., zymosan, curdlan), background of the mice (C56BL6, 129/Sv) and human or mouse cells. In addition, as discussed above, $\beta 1,3-$ glucan exposure and whether chitin or mannans are masking the $\beta 1,3$-glucans could all help to determine whether Dectin- 1 is involved in any particular experimental setup. Furthermore, the role of additional receptors such as Dectin-2, Mannose Receptor, TLR2 and TLR4 likely differs due to receptor expression on different cell types, exposure of the relevant ligands on different Candida spp. and interaction with the other Candida-interacting receptors on each particular cell type (Netea et al., 2006; Hall et al., 2013; Ifrim et al., 2014, 2016; Estrada-Mata et al., 2015; Perez-Garcia et al., 2016). When the Candida spp. were killed with thimerosal, we observed much lower levels of cytokine production from BMDMs than with live Candida spp. and we did not observe any significant Dectin-1 dependence. While thimerosal maintains cell wall integrity (Hall et al., 2013), the
Candida cells are then unable to undergo any changes due to different growth media, encountering host immune cells or differences in oxygen levels that may alter $\beta 1,3$-glucan exposure or masking. Various studies have previously found increased responses to heat killed $C$. albicans and major dependence on Dectin-1 in response to heat killed $C$. albicans, compared to live or thimerosal killed $C$. albicans, as this exposes $\beta 1,3$-glucans (Gow et al., 2007; Hall et al., 2013; Estrada-Mata et al., 2015). Without increased exposure of $\beta 1,3$-glucans either due to heat killing or due to different culture conditions, the thimerosal killed Candida spp. may not be able to induce major Dectin-1 dependent responses. Overall, we found that different cytokine profiles are produced dependent on cell type, Candida spp. and/or strain, and Candida cell viability.

In addition to mediating innate myeloid cell cytokine responses, we found that in some cases effector $\mathrm{T}$ cell responses are enhanced in cells from Dectin-1 KO mice. Previous studies have shown that following antigen restimulation splenocytes from Dectin-1 KO mice displayed reduced IFN- $\gamma$ and/or IL17 production, however, these studies used heat-killed or UVinactivated Candida spp. (Robinson et al., 2009; Chen et al., 2017). As heat-killing and UV-inactivation alters the fungal cell wall and exposes different ligands thereby increasing the dependence on Dectin-1 (Gow et al., 2007; Estrada-Mata et al., 2015), this could potentially explain the difference in our results. Our data demonstrate an enhanced generic and antigen-specific Th17 response (albicans and parapsilosis) and an enhanced antigen-specific IFN- $\gamma$ response (albicans) in Dectin-1 KO mice, likely due to increased exposure of $\mathrm{T}$ cells to Candida in vivo due to the inability to control these infections. However, the fungal burden is increased in Dectin-1 KO mice during infection with each of the Candida spp. while IL-17 responses are not increased in response to C. glabrata or C. tropicalis. Therefore, in addition to increased fungal burden it is possible that Th17 promoting cytokines such as TGF- $\beta$, IL- 6 and IL-23 are differentially regulated in vivo in response to the various Candida spp. (Sandquist and Kolls, 2018). In support of this theory, serum levels of IL-6 are increased in Dectin-1 KO mice during infection with $C$. albicans, and cells from these mice demonstrate the highest Candida-induced IL-17 response. It is also possible that the enhanced Th17 effector response may at least in part be mediated by Dectin- 2 as Dectin- 2 has been shown to induce Th17 responses to C. albicans (Saijo et al., 2010). The enhanced Th17 response in Dectin-1 KO cells could potentially be a compensatory mechanism by Dectin-2 to deal with the lack of a Dectin-1 response. Thus Dectin-1 is important for both innate and adaptive immune responses to multiple Candida spp. although it's role in specific responses varies with different Candida spp.

\section{CONCLUSION}

The present study shows that multiple clinically relevant Candida spp. induce significantly different innate cytokine profiles and that Dectin-1 mediates these cytokine responses in a cell type and Candida spp. dependent manner, indicating that results 
with C. albicans cannot necessarily be fully extrapolated to other Candida spp.

\section{DATA AVAILABILITY}

All datasets generated for this study are included in the manuscript and/or the Supplementary Files.

\section{AUTHOR CONTRIBUTIONS}

AT designed and performed the experiments and wrote the manuscript. JG, LW, DF, and KL performed the experiments. PT and NG guided the research. SO conceptualized and guided the research, designed and performed the experiments, and wrote the manuscript. All authors contributed to the manuscript revision, and read and approved the submitted version.

\section{REFERENCES}

Alangaden, G. J. (2011). Nosocomial fungal infections: epidemiology, infection control, and prevention. Infect. Dis. Clin. North Am. 25, 201-225. doi: 10.1016/ j.idc. 2010.11 .003

Anaissie, E., Hachem, R., K-Tin-U, C., Stephens, L. C., and Bodey, G. P. (1993). Experimental hematogenous candidiasis caused by Candida krusei and Candida albicans: species differences in pathogenicity. Infect. Immun. 61, 1268-1271.

Arendrup, M., Horn, T., and Frimodt-Moller, N. (2002). In vivo pathogenicity of eight medically relevant Candida species in an animal model. Infection 30, 286-291. doi: 10.1007/s15010-002-2131-0

Ballou, E. R., Avelar, G. M., Childers, D. S., Mackie, J., Bain, J. M., Wagener, J., et al. (2016). Lactate signalling regulates fungal beta-glucan masking and immune evasion. Nat. Microbiol. 2:16238. doi: 10.1038/nmicrobiol.2016.238

Branzk, N., Lubojemska, A., Hardison, S. E., Wang, Q., Gutierrez, M. G., Brown, G. D., et al. (2014). Neutrophils sense microbe size and selectively release neutrophil extracellular traps in response to large pathogens. Nat. Immunol. 15, 1017-1025. doi: 10.1038/ni.2987

Brieland, J., Essig, D., Jackson, C., Frank, D., Loebenberg, D., Menzel, F., et al. (2001). Comparison of pathogenesis and host immune responses to Candida glabrata and Candida albicans in systemically infected immunocompetent mice. Infect. Immun. 69, 5046-5055. doi: 10.1128/iai.69.8.5046-5055.2001

Brown, G. D., Denning, D. W., Gow, N. A., Levitz, S. M., Netea, M. G., and White, T. C. (2012). Hidden killers: human fungal infections. Sci. Transl. Med. 4:165rv13. doi: 10.1126/scitranslmed.3004404

Brown, G. D., and Gordon, S. (2001). Immune recognition. A new receptor for beta-glucans. Nature 413, 36-37.

Chen, S. M., Shen, H., Zhang, T., Huang, X., Liu, X. Q., Guo, S. Y., et al. (2017). Dectin-1 plays an important role in host defense against systemic Candida glabrata infection. Virulence 8, 1643-1656. doi: 10.1080/21505594. 2017.1346756

Chowdhary, A., Sharma, C., and Meis, J. F. (2017). Candida auris: a rapidly emerging cause of hospital-acquired multidrug-resistant fungal infections globally. PLoS Pathog. 13:e1006290. doi: 10.1371/journal.ppat.1006290

Duan, Z., Chen, Q., Zeng, R., Du, L., Liu, C., Chen, X., et al. (2018). Candida tropicalis induces pro-inflammatory cytokine production, NF-kappaB and MAPKs pathways regulation, and dectin-1 activation. Can. J. Microbiol. 64, 937-944. doi: 10.1139/cjm-2017-0559

Estrada-Mata, E., Navarro-Arias, M. J., Perez-Garcia, L. A., Mellado-Mojica, E., Lopez, M. G., Csonka, K., et al. (2015). Members of the Candida parapsilosis complex and Candida albicans are differentially recognized by human peripheral blood mononuclear cells. Front. Microbiol. 6:1527. doi: 10 . 3389/fmicb.2015.01527

\section{FUNDING}

SO was funded by a Sir Henry Dale Fellowship jointly funded by the Wellcome Trust and the Royal Society (Grant Number 099953/Z/12/Z) and by a Wellcome Trust Cross-Disciplinary Award. PT is supported by a Wellcome Trust Investigator Award (107964/Z/15/Z) and the UK Dementia Research Institute. NG was supported by the Wellcome Trust Investigator, Collaborative, Equipment, Strategic and Biomedical Resource awards (086827, $075470,097377,101873,200208,093378$, and 099197), and by the MRC Centre for Medical Mycology (N006364/1).

\section{SUPPLEMENTARY MATERIAL}

The Supplementary Material for this article can be found online at: https://www.frontiersin.org/articles/10.3389/fmicb. 2019.01800/full\#supplementary-material

Fidel, P. L. Jr., Vazquez, J. A., and Sobel, J. D. (1999). Candida glabrata: review of epidemiology, pathogenesis, and clinical disease with comparison to C. albicans. Clin. Microbiol. Rev. 12, 80-96. doi: 10.1128/cmr.12.1.80

Gow, N. A., Netea, M. G., Munro, C. A., Ferwerda, G., Bates, S., Mora-Montes, H. M., et al. (2007). Immune recognition of Candida albicans beta-glucan by dectin-1. J. Infect. Dis. 196, 1565-1571.

Gow, N. A., van de Veerdonk, F. L., Brown, A. J., and Netea, M. G. (2011). Candida albicans morphogenesis and host defence: discriminating invasion from colonization. Nat. Rev. Microbiol. 10, 112-122. doi: 10.1038/nrmicro2711

Hall, R. A., Bates, S., Lenardon, M. D., Maccallum, D. M., Wagener, J., Lowman, D. W., et al. (2013). The Mnn2 mannosyltransferase family modulates mannoprotein fibril length, immune recognition and virulence of Candida albicans. PLoS Pathog. 9:e1003276. doi: 10.1371/journal.ppat.1003276

Hidron, A. I., Edwards, J. R., Patel, J., Horan, T. C., Sievert, D. M., Pollock, D. A., et al. (2008). NHSN annual update: antimicrobial-resistant pathogens associated with healthcare-associated infections: annual summary of data reported to the national healthcare safety network at the centers for disease control and prevention, 2006-2007. Infect. Control Hosp. Epidemiol. 29, 996-1011. doi: 10.1086/591861

Ifrim, D. C., Bain, J. M., Reid, D. M., Oosting, M., Verschueren, I., Gow, N. A., et al. (2014). Role of Dectin-2 for host defense against systemic infection with Candida glabrata. Infect. Immun. 82, 1064-1073. doi: 10.1128/IAI.01189-13

Ifrim, D. C., Quintin, J., Courjol, F., Verschueren, I., van Krieken, J. H., Koentgen, F., et al. (2016). The role of dectin-2 for host defense against disseminated candidiasis. J. Interferon Cytokine Res. 36, 267-276. doi: 10.1089/jir.2015.0040

Karacaer, Z., Oncul, O., Turhan, V., Gorenek, L., and Ozyurt, M. (2014). A surveillance of nosocomial candida infections: epidemiology and influences on mortalty in intensive care units. Pan Afr. Med. J. 19:398. doi: 10.11604/pamj. 2014.19.398.4960

LeibundGut-Landmann, S., Gross, O., Robinson, M. J., Osorio, F., Slack, E. C., Tsoni, S. V., et al. (2007). Syk- and CARD9-dependent coupling of innate immunity to the induction of T helper cells that produce interleukin 17. Nat. Immunol. 8, 630-638. doi: 10.1038/ni1460

Linden, J. R., Maccani, M. A., Laforce-Nesbitt, S. S., and Bliss, J. M. (2010). High efficiency opsonin-independent phagocytosis of Candida parapsilosis by human neutrophils. Med. Mycol. 48, 355-364. doi: 10.1080/13693780903164566

Lo, H. J., Kohler, J. R., DiDomenico, B., Loebenberg, D., Cacciapuoti, A., and Fink, G. R. (1997). Nonfilamentous C. albicans mutants are avirulent. Cell 90, 939-949. doi: 10.1016/s0092-8674(00)80358-x

Lopes, J. P., Stylianou, M., Backman, E., Holmberg, S., Jass, J., Claesson, R., et al. (2018). Evasion of immune surveillance in low oxygen environments enhances Candida albicans Virulence. mBio 9:e2120-18. doi: 10.1128/mBio.02120-18

Marakalala, M. J., Vautier, S., Potrykus, J., Walker, L. A., Shepardson, K. M., Hopke, A., et al. (2013). Differential adaptation of Candida albicans in vivo 
modulates immune recognition by dectin-1. PLoS Pathog. 9:e1003315. doi: 10.1371/journal.ppat.1003315

Netea, M. G., Gow, N. A., Munro, C. A., Bates, S., Collins, C., Ferwerda, G., et al. (2006). Immune sensing of Candida albicans requires cooperative recognition of mannans and glucans by lectin and Toll-like receptors. J. Clin. Invest. 116, 1642-1650. doi: 10.1172/jci27114

Patin, E. C., Jones, A. V., Thompson, A., Clement, M., Liao, C. T., Griffiths, J. S., et al. (2016). IL-27 Induced by Select Candida spp. via TLR7/NOD2 Signaling and IFN-beta production inhibits fungal clearance. J. Immunol. 197, 208-221. doi: 10.4049/jimmunol.1501204

Patin, E. C., Thompson, A., and Orr, S. J. (2018). Pattern recognition receptors in fungal immunity. Semin. Cell Dev. Biol. 89, 24-33. doi: 10.1016/j.semcdb.2018. 03.003

Perez-Garcia, L. A., Csonka, K., Flores-Carreon, A., Estrada-Mata, E., MelladoMojica, E., Nemeth, T., et al. (2016). Role of protein glycosylation in Candida parapsilosis cell wall integrity and host interaction. Front. Microbiol. 7:306. doi: 10.3389/fmicb.2016.00306

Robinson, M. J., Osorio, F., Rosas, M., Freitas, R. P., Schweighoffer, E., Gross, O., et al. (2009). Dectin-2 is a syk-coupled pattern recognition receptor crucial for Th17 responses to fungal infection. J. Exp. Med. 206, 2037-2051. doi: 10.1084/ jem.20082818

Saijo, S., Fujikado, N., Furuta, T., Chung, S. H., Kotaki, H., Seki, K., et al. (2007). Dectin-1 is required for host defense against Pneumocystis carinii but not against Candida albicans. Nat. Immunol. 8, 39-46. doi: 10.1038/ni1425

Saijo, S., Ikeda, S., Yamabe, K., Kakuta, S., Ishigame, H., Akitsu, A., et al. (2010). Dectin-2 recognition of alpha-mannans and induction of Th17 cell differentiation is essential for host defense against Candida albicans. Immunity 32, 681-691. doi: 10.1016/j.immuni.2010.05.001

Sandquist, I., and Kolls, J. (2018). Update on regulation and effector functions of Th17 cells. F1000Research 7:205. doi: 10.12688/f1000research.13020.1
Sem, X., Le, G. T., Tan, A. S., Tso, G., Yurieva, M., Liao, W. W., et al. (2016). Beta-glucan exposure on the fungal cell wall tightly correlates with competitive fitness of Candida Species in the mouse gastrointestinal tract. Front. Cell. Infect. Microbiol. 6:186. doi: 10.3389/fcimb.2016.00186

Silva, S., Negri, M., Henriques, M., Oliveira, R., Williams, D. W., and Azeredo, J. (2012). Candida glabrata, Candida parapsilosis and Candida tropicalis: biology, epidemiology, pathogenicity and antifungal resistance. FEMS Microbiol. Rev. 36, 288-305. doi: 10.1111/j.1574-6976.2011.00278.x

Taylor, P. R., Tsoni, S. V., Willment, J. A., Dennehy, K. M., Rosas, M., Findon, H., et al. (2007). Dectin-1 is required for beta-glucan recognition and control of fungal infection. Nat. Immunol. 8, 31-38. doi: 10.1038/ni1408

Toth, A., Csonka, K., Jacobs, C., Vagvolgyi, C., Nosanchuk, J. D., Netea, M. G., et al. (2013). Candida albicans and Candida parapsilosis induce different T-cell responses in human peripheral blood mononuclear cells. J. Infect. Dis. 208, 690-698. doi: 10.1093/infdis/jit188

Trofa, D., Gacser, A., and Nosanchuk, J. D. (2008). Candida parapsilosis, an emerging fungal pathogen. Clin. Microbiol. Rev. 21, 606-625. doi: 10.1128/ CMR.00013-08

Conflict of Interest Statement: The authors declare that the research was conducted in the absence of any commercial or financial relationships that could be construed as a potential conflict of interest.

Copyright (C) 2019 Thompson, Griffiths, Walker, da Fonseca, Lee, Taylor, Gow and Orr. This is an open-access article distributed under the terms of the Creative Commons Attribution License (CC BY). The use, distribution or reproduction in other forums is permitted, provided the original author(s) and the copyright owner(s) are credited and that the original publication in this journal is cited, in accordance with accepted academic practice. No use, distribution or reproduction is permitted which does not comply with these terms. 